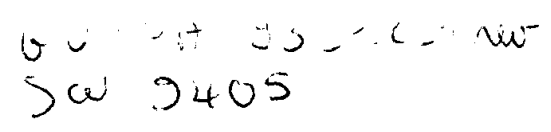

GUTPA 93/12/1

Revised version January 1994

\title{
Graviton Creation in an Expanding Universe
}

\author{
R G Moorhouse \\ Department of Physics and Astronomy, University of Glasgow \\ Glasgow G12 8QQ, United Kingdom
}

\section{A B Henriques and L E Mendes}

Departamento de Fisica, Instituto Superior Técnico,

Av. Rovisco Pais, 1096 Lisboa-Codex, Portugal

\begin{abstract}
PQDQEQ5? 1

In graviton creation a new derivation is given of a differential equation for Bogoliubov coefficients, defined as continuous functions of time. The resulting method for graviton production is applicable for any wavelengths and for a Friedmann universe scale factor which is an arbitrary function of time; in those particular cases where the transitions between cosmic eras can be treated as sudden, so that the well known discrete Bogoliubov coefficients can alternatively be used, the two results agree. The relation to the result for gravitational wave production through amplitude amplification for classical metric perturbations is given explicitly. The method is also applicable to theories with BransDicke type scalars such as extended inflation.
\end{abstract}

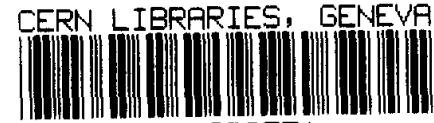


The origin of hypothetical relic gravitons from the early universe is in quantum fluctuations of the space-time background. When the comoving wavelength is greater than $\mathrm{H}^{-1}$ (where $\mathrm{H}$ is the Hubble parameter) these fluctuations, regarded as classical gravitational waves, can amplify in an expanding universe [1]. Most commonly, following this viewpoint, the calculation of relic gravitational waves has been done by relating the quantum mechanical fluctuation to a statistical average of an ensemble of classical fields [2]. An alternative, purely quantum mechanical, viewpoint is to consider the changes in the graviton creation and annihilation operators as the universe progresses, say, from an inflation era through a radiation era to a matter era. Regarding the transitions from one era to another as sudden, the changes can be found by calculating the discrete Bogoliubov coefficients at the transitions and then the resulting gravitons can be found by using the combined Bogoliubov coefficients [3,4]. However there is yet another purely quantum mechanical viewpoint in which Bogoliubov coefficients are defined and calculated as continuous functions of time, and the gravitons (gravitational waves) at any time are given in terms of the Bogoliubov coefficients at that time. This is the viewpoint developed in this paper, with implicit use of the Heisenberg picture.

The production of particles in an expanding universe using a formalism of continuously evolving Bogoliubov coefficients was first investigated by Parker[5], who derived integral equations in the coefficients, involving an essentially arbitrary function $W(k, \tau)$, where $k$ is the wave number and $\tau$ the conformal time. In this paper we derive the equivalent differential equations, in the case of gravitational waves, by a new and different method in which for physical reasons $\mathrm{W}$ turns out to be of the form $\mathrm{W}=\mathrm{k}$. (Such a restriction has previously been considered for other reasons[6]). The resulting equations take a rather simple form and are amenable in some important cases to analytic solution. The method can be simply extended to theories with scalars such as Brans-Dicke theory and extended inflation.

Consider a cosmic period described by a Friedmann-Robertson-Walker metric $\mathrm{a}^{2}(\tau)\left(-\mathrm{d} \tau^{2}+\right.$ $\underline{\mathrm{dx}} \cdot \underline{\mathrm{dx}})$. We visualize the arbitrary scale factor $\mathrm{a}(\tau)$ as a curve in the a, $\tau$ plane. We split the curve into segments and approximate each segment by a straight line chord (as shown in the figure):

$$
S(\tau)=B(\tau-b)
$$

If the chord and segment start at $\left(a_{i}, \tau_{i}\right)$ and end at $\left(a_{f}, \tau_{f}\right)$ then

$$
B=\left(a_{f}-a_{i}\right) /\left(\tau_{f}-\tau_{i}\right) \quad b=\tau_{i}-a_{i} / B=\tau_{f}-a_{f} / B
$$

Now suppose the physical system to proceed by way of the chord rather than the segment and for the chord under consideration let $\alpha, \beta$ be Bogoliubov coefficients in the following sense. The metric perturbation in the period $\tau_{\mathrm{i}}$ to $\tau_{\mathrm{f}}$ is $[3,4]$ : 


$$
\begin{aligned}
& \mathrm{h}_{\mathrm{ij}}=\sqrt{8 \pi \mathrm{G}} \sum_{\lambda=1}^{2} \int \frac{\mathrm{d}^{3} \mathrm{k}}{(2 \pi)^{3 / 2} \mathrm{~S}(\tau) \sqrt{2 \mathrm{k}}} \mu(\underline{\mathrm{k}}, \tau) \\
& \mu(\underline{\mathrm{k}} \tau)=\left[\mathrm{a}_{\lambda}(\underline{\mathrm{k}}) \varepsilon_{\mathrm{ij}}(\underline{\mathrm{k}}, \lambda) \mathrm{e}^{\mathrm{ik} \cdot \underline{\mathrm{x}}} \xi(\mathrm{k}, \tau)+\text { herm.conj. }\right]
\end{aligned}
$$

where $\varepsilon_{\mathrm{ij}}(\underline{\mathrm{k}}, \lambda)$ are two polarization tensors, $\mathrm{a}_{\lambda}(\underline{\mathrm{k}})$ is the corresponding annihilation operator and $\xi(\mathrm{k} \tau)$ is the mode function for the gravitational waves, obeying the corresponding gravitational wave equation [4]

$$
\xi^{\prime \prime}+\left(\mathrm{k}^{2}-\mathrm{S}^{\prime \prime} / \mathrm{S}\right) \xi=0
$$

where (1) implies $S " / S=0$. The annihilation and creation operators in (4) are expressed in terms of time fixed annihilation and creation operators, $A(\underline{k})$ and $A^{\dagger}(\underline{k})$, through the Bogoliubov coefficients $\alpha$ and $\beta$ by

$$
\mathrm{a}(\underline{\mathrm{k}})=\alpha \mathrm{A}(\underline{\mathrm{k}})+\beta^{*} \mathrm{~A}^{\dagger}(-\underline{\mathrm{k}})
$$

The $\mathrm{A}(\mathrm{k})$ could, for example, be the annihilation operators corresponding to the gravitational wave modes in some previous radiation era.

Now consider the system evolving along the chords which approximate the curve with instantaneous transitions at the junctions of the chords. Consider two neighbouring chords denoted by 1 and 2 with junction at $\tau=\eta$. We find the change in the Bogoliubov coefficients from chord 1 to chord 2 by requiring that $h_{i j}$ and its first derivative be continuous at $\tau=\eta$.

$$
\begin{aligned}
& \alpha_{1} \frac{\xi_{1}}{S_{1}}+\beta_{1} \frac{\xi_{1}^{*}}{S_{1}}=\alpha_{2} \frac{\xi_{2}}{S_{2}}+\beta_{2} \frac{\xi_{2}^{*}}{S_{2}} \\
& \alpha_{1}\left(\frac{\xi_{1}^{\prime}}{S_{1}}-\frac{S_{1}^{\prime}}{S_{1}^{2}} \xi_{1}\right)+\beta_{1}\left(\frac{\xi_{1}^{*}}{S_{1}}-\frac{S_{1}^{\prime}}{\left.S_{1}^{2} \xi_{1}^{*}\right)=}\right. \\
& \alpha_{2}\left(\frac{\xi_{2}^{\prime}}{S_{2}}-\frac{S_{2}^{\prime}}{S_{2}^{2}} \xi_{2}\right)+\beta_{2}\left(\frac{\xi_{2}^{*}}{S_{2}}-\frac{S_{2}^{\prime}}{S_{2}^{2}} \xi_{2}^{*}\right)
\end{aligned}
$$

Using eqns (1) and (2) where $S_{1}=B_{1}\left(\tau-b_{1}\right), S_{2}=B_{2}\left(\tau-b_{2}\right)$ and $S_{1}(\eta)=S_{2}(\eta)$ eqns (7) 
and (8) give at $\tau=\eta$.

$$
\begin{aligned}
& \alpha_{1} \xi_{1}+\beta_{1} \xi_{1}^{*}=\alpha_{2} \xi_{2}+\beta_{2} \xi_{2}^{*} \\
& \alpha_{1} \xi_{1}^{\prime}+\beta_{1} \xi_{1}^{*^{\prime}}+\rho\left(\alpha_{1} \xi_{1}+\beta_{1} \xi_{1}^{*}\right)=\alpha_{2} \xi_{2}+\beta_{2} \xi_{2}^{*}
\end{aligned}
$$

where $\rho=\frac{1}{\eta-b_{2}}-\frac{1}{\eta-b_{1}}$

The mode functions $\xi$ are solutions of eqn (5) where $\mathrm{S}_{1}{ }^{\prime \prime} / \mathrm{S}_{1}=\mathrm{S}_{2}{ }^{\prime \prime} / \mathrm{S}_{2}=0$; we take the solution

$$
\xi=\mathrm{e}^{-\mathrm{ik}\left(\tau-\tau_{0}\right)}
$$

with $\tau_{0}$ the same arbitrary constant in all chords. Eqns (9) and (10) can then be solved to give

$$
\begin{aligned}
& \alpha_{2}=\alpha_{1}+\frac{\mathrm{i}}{2 \mathrm{k}}\left(\alpha_{1}+\beta_{1} \mathrm{e}^{2 \mathrm{ik}\left(\tau-\tau_{0}\right)}\right) \rho \\
& \beta_{2}=\beta_{1}-\frac{\mathrm{i}}{2 \mathrm{k}}\left(\beta_{1}+\alpha_{1} \mathrm{e}^{-2 \mathrm{ik}\left(\tau-\tau_{0}\right)}\right) \rho
\end{aligned}
$$

At this stage we may note that the method of continuity of $h_{i j}$ and $h^{\prime}{ }_{i j}$ is that expounded by Abbott and Harari [4], (hereafter referred to as AH) for finding the Bogoliubov coefficients in a sudden change from one expansion mode to another; but they take the physical situation that $S_{1}^{\prime}=S_{2}^{\prime}, \xi_{1}^{\prime} \neq \xi_{2}^{\prime}$ whereas we have $S_{1}^{\prime} \neq S_{2}^{\prime}$ and $\xi_{1}^{\prime}=\xi_{2}^{\prime}$. We shall see below however that our method is to take the appropriate limit and then we reproduce precisely the results of $\mathrm{AH}$ in their type of physical scenario.

In equations (13 and (14) it remains to evaluate $\rho$ in terms of the curve a( $\tau$ ); preparing to take the limit of small segments we do this to first order in $\Delta \tau$, where $\Delta \tau$ is the time interval between the midpoints of the adjoining segments. From eqns (2) and (11)

$$
\rho=\frac{b_{2}-b_{1}}{\left(\eta-b_{2}\right)\left(\eta-b_{1}\right)}=\frac{a(\eta)\left(B_{2}-B_{1}\right)}{a^{2}(\eta)}=\frac{a_{2}^{\prime}-a_{1}^{\prime}}{a(\eta)}=\frac{a^{\prime \prime}}{a} \Delta \tau
$$

In the limit that the segments tend to zero we thus obtain from (13) and (14) the differential equations 


$$
\begin{aligned}
& \alpha^{\prime}(\tau)=\frac{i}{2 k}\left(\alpha(\tau)+\beta(\tau) e^{2 i k\left(\tau-\tau_{0}\right)}\right) \frac{a^{\prime \prime}(\tau)}{a(\tau)} \\
& \beta^{\prime}(\tau)=-\frac{i}{2 k}\left(\beta(\tau)+\alpha(\tau) e^{-2 i k\left(\tau-\tau_{0}\right)}\right) \frac{a^{\prime \prime}(\tau)}{a(\tau)}
\end{aligned}
$$

Before discussing the solution of these equations we should make the connection with the method of Parker [5]; we do this by re-expressing that method in our more simple formalism:

In the era where the scale factor is $a(\tau)$

$$
\mathrm{h}_{\mathrm{ij}}=\sqrt{8 \pi \mathrm{G}} \sum_{\lambda=1}^{2} \int \frac{\mathrm{d}^{3} \mathrm{k}}{(2 \pi)^{3 / 2} \mathrm{a}(\tau) \sqrt{2 \mathrm{~W}(\mathrm{k}, \tau)}} \bar{\mu}(\mathrm{k}, \tau)
$$

$\bar{\mu}(\mathrm{k} \tau)=\left[\sum \mathrm{a}_{\lambda}(\mathrm{k}, \tau) \varepsilon_{\mathrm{ij}}(\underline{\mathrm{k}}, \lambda) \mathrm{e}^{\mathrm{ik} \cdot \mathrm{x}} \exp \left(-\mathrm{i} \int_{\tau_{\mathrm{o}}}^{\tau} \mathrm{W}\left(\mathrm{k}, \tau^{\prime}\right) \mathrm{d} \tau^{\prime}\right)+\right.$ herm. conj $]$

where $\mathrm{W}(\mathrm{k}, \tau)$ is essentially arbitrary and where $\mathrm{a}_{\lambda}(\underline{\mathrm{k}}, \tau)$ are time dependent annihilation operators expressed through Bogoliubov coefficients as

$$
\mathrm{a}(\underline{\mathrm{k}}, \tau)=\alpha(\tau) \mathrm{A}(\underline{\mathrm{k}})+\beta^{*}(\tau) \mathrm{A}^{\dagger}(-\underline{\mathrm{k}})
$$

If, as proposed in reference 6 , we now make the simple ansatz $\mathrm{W}=\mathrm{k}$, then

$$
\bar{\mu}^{\prime \prime}+\left(\mathrm{k}^{2}-\frac{\mathrm{a}^{\prime \prime}}{\mathrm{a}}\right) \bar{\mu}=0
$$

Then the method of Green's functions as used by Parker [5], with $\alpha\left(\tau_{0}\right)=1, \beta\left(\tau_{0}\right)=0$, yields the integral equations

$$
\alpha(\tau)=1+\frac{\mathrm{i}}{2 \mathrm{k}} \int_{\tau_{0}}^{\tau} \mathrm{d} \tau^{\prime}\left(\alpha\left(\tau^{\prime}\right)+\beta\left(\tau^{\prime}\right) \mathrm{e}^{2 \mathrm{ik}\left(\tau^{\prime}-\tau_{0}\right)}\right) \frac{\mathrm{a}^{\prime \prime}\left(\tau^{\prime}\right)}{\mathrm{a}\left(\tau^{\prime}\right)}
$$




$$
\beta(\tau)=-\frac{i}{2 k} \int_{\tau_{0}}^{\tau} d \tau^{\prime}\left(\beta\left(\tau^{\prime}\right)+\alpha\left(\tau^{\prime}\right) e^{-2 i k\left(\tau^{\prime}-\tau_{0}\right)}\right) \frac{a^{\prime \prime}\left(\tau^{\prime}\right)}{a(\tau)}
$$

and the differential form of these equations is just our equations (16) and (17) above. In our method of derivation through segmentation the choice of straight line chords $S=B(\tau-b)$ leading to the mode functions $\xi \propto \mathrm{e}^{-\mathrm{ik} \tau}$, was partly motivated by the fact that this corresponds to a radiation dominated universe where conformal invariance gives zero gravitational wave production during the universe's travel along the chord, but production only at the junctions. This choice of chord is of course not necessary, as can be seen from the methods of $\mathrm{AH}$, but it is certainly the simplest.

The solution of equations (16) and (17) is simplest through the substitution

$$
\begin{aligned}
& \mathrm{X}=\alpha(\tau) \mathrm{e}^{-\mathrm{ik}\left(\tau-\tau_{0}\right)}+\beta(\tau) \mathrm{e}^{\mathrm{ik}\left(\tau-\tau_{0}\right)} \\
& \mathrm{Y}=\alpha(\tau) \mathrm{e}^{-\mathrm{ik}\left(\tau-\tau_{0}\right)}-\beta(\tau) \mathrm{e}^{\mathrm{ik}\left(\tau-\tau_{0}\right)}
\end{aligned}
$$

leading to

$$
\begin{aligned}
& X^{\prime \prime}+\left(k^{2}-a^{\prime \prime} / a\right) \quad X=0 \\
& Y=i X^{\prime} / k
\end{aligned}
$$

For a matter dominated universe or for exponential inflation

$$
\mathrm{a}^{\prime \prime} / \mathrm{a}=2 / \tau^{2}
$$

and the solution of (26) is simple. We find the two independent solutions, with $x=k \tau$,

$$
\begin{array}{ll}
\alpha_{1}(\mathrm{x})=\left(1-\mathrm{i} / \mathrm{x}-1 / 2 \mathrm{x}^{2}\right) & \beta_{1}(\mathrm{x})=\mathrm{e}^{-2 \mathrm{i}\left(\mathrm{x}-\mathrm{x}_{\mathrm{O}}\right)} / 2 \mathrm{x}^{2} \\
\alpha_{2}(\mathrm{x})=\mathrm{e}^{2 \mathrm{i}\left(\mathrm{x}-\mathrm{x}_{0}\right)} / 2 \mathrm{x}^{2} & \beta_{2}(\mathrm{x})=\left(1+\mathrm{i} / \mathrm{x}-1 / 2 \mathrm{x}^{2}\right)
\end{array}
$$

The general solution is a linear superposition of these, the coefficients being chosen so as to satisfy boundary conditions. We can illustrate the solution, and at the same time compare with the results in $\mathrm{AH}$ by giving the solution for an exponential inflationary era, beginning at $\tau_{1}$ and ending at $\tau_{2}$, immediately preceded and followed by radiation eras. For precise comparison we need to impose the same junction conditions as in $\mathrm{AH}$, to wit that $\mathrm{a}(\tau)$ and $\mathrm{a}^{\prime}(\tau)$ be continuous at the junctions between inflation and radiation. If we make the choice $\tau_{\mathrm{o}}=-2 \tau_{2}$ then the mode function $\xi$ at the end of inflation takes the value $\mathrm{e}^{-\mathrm{ik} \tau_{2}}$ and is continuous with the 
mode function chosen in $\mathrm{AH}$ for the final radiation era, ensuring an identical phase convention for the Bogoliubov coefficients at the beginning of that era.

The general solution of the differential equations (16) (17) is

$$
\begin{aligned}
& \alpha=c_{1} \alpha_{1}(x)+c_{2} \alpha_{2}(x) \\
& \beta=c_{1} \beta_{1}(x)+c_{2} \beta_{2}(x)
\end{aligned}
$$

and choosing $\beta=0$ at $x=x_{1},\left(\tau=\tau_{1}\right)$ gives

$$
c_{1}=c\left(1+i / x_{1}-1 / 2 x_{1}^{2}\right) \quad c_{2}=-c e^{-2 i\left(x_{1}-2 x_{2}\right)} / 2 x_{1}^{2} .
$$

$\beta=0$ implies $|\alpha|=1$. Also, in the method of $\mathrm{AH}, \alpha=1$ at the end of the preceding radiation era with the multiplying mode function value being $\mathrm{e}^{\mathrm{ix}}{ }_{1}$; to agree with this we require in our calculation that $\alpha=e^{2 \mathrm{i}\left(\mathrm{x}_{1}-\mathrm{x}_{2}\right)}$ at the beginning of inflation and then (31) and (33) give

$$
c=e^{2 i\left(x_{1}-x_{2}\right)}
$$

The values of $\alpha$ and $\beta$ generated in the inflationary period through the differential equations (16) and (17) are then given by (31), (33) and (34) as

$$
\begin{aligned}
& \alpha=e^{2 i\left(x_{1}-x_{2}\right)}\left(1+\frac{i}{x_{1}}-\frac{1}{2 x_{1}^{2}}\right)\left(1-\frac{i}{x_{2}}-\frac{1}{2 x_{2}^{2}}\right)-\frac{1}{4 x_{1}^{2} x_{2}^{2}} \\
& \beta=e^{2 i x_{1}}\left(1+\frac{i}{x_{1}}-\frac{1}{2 x_{1}^{2}}\right) \frac{1}{2 x_{2}^{2}}-e^{2 i x_{2}}\left(1+\frac{i}{x_{2}}-\frac{1}{2 x_{2}^{2}}\right) \frac{1}{2 x_{1}^{2}}
\end{aligned}
$$

We now apply the method of $\mathrm{AH}$, where the Bogoliubov coefficients are generated only at the beginning and at the end of the inflationary era. We find at the beginning and end respectively

$$
\begin{array}{ll}
\alpha_{i}=e^{2 i x_{1}}\left(1+\frac{i}{x_{1}}-\frac{1}{2 x_{1}^{2}}\right) & \beta_{i}=-\frac{1}{2 x_{1}^{2}} \\
\alpha_{f}=e^{-2 i x} 2\left(1-\frac{i}{x_{2}}-\frac{1}{2 x_{2}^{2}}\right) & \beta_{f}=\frac{1}{2 x_{2}^{2}}
\end{array}
$$

The total $\alpha$ and $\beta$ generated are given by the law of composition of Bogoliubov coefficients as 
$\alpha_{\mathrm{i}} \alpha_{\mathrm{f}}+\beta_{\mathrm{i}} \beta_{\mathrm{f}}{ }^{*}$ and $\beta_{\mathrm{i}} \alpha_{\mathrm{f}}^{*}+\beta_{\mathrm{f}} \alpha_{\mathrm{i}}$. They agree precisely with equations (35) and (36). Thus, as we should expect, we find agreement between the two methods in a model case where both should give the exact answer.

Though the method propounded in this paper does not solve any fundamental problems and is rather to be regarded as a calculational tool, as such it does offer advantages over the method of sudden transitions $[3,4]$ and has no disadvantages with respect to other methods $[2,7-11]$ used to calculate graviton or gravitational wave production. We can in principle integrate eqn (26) for any arbitrary expansion factor a $(\tau)$. In particular we can calculate through either sudden or gradual transtions and the particular value of $\mathrm{k}=2 \pi \mathrm{a} / \lambda$ is not a limitation. For any given value of $\mathrm{k}$ the calculation can be done continuously right through all eras up to some time in the matter era.

However the final stage does require some discussion. If we are interested in the production of gravitational waves into say the last radiation era, then as we have seen above in the comparison with the paper of $\mathrm{AH}$ there is no extra graviton production at the beginning of this era: this is because (following our procedure) in the preradiation era the final segment (and chord) have the same slope and mode function as the succeeding radiation era. For production of gravitational waves into the matter era the situation is different. Up to any arbitrary time say $\tau_{D}$ in the matter era ( $\tau_{D}$ at or before the time of interest) we can continue to calculate graviton production through equations (16) and (17), with latterly a $(\tau)$ being the matter era scale factor. Then just before $\tau_{\mathrm{D}}$, the final chord of our segmental curve will be continuous in slope with the succeeding matter curve; but it will have a radiation type mode function $\left(\xi \propto \mathrm{e}^{-\mathrm{ik}\left(\tau-\tau_{0}\right)}\right)$ while after $\tau_{D}$ we require a matter era type mode function of the type $\xi_{m}=e^{-i k \tau}(1-i / k \tau)$. This is a change in the definition of graviton creation and annihilation operators, and gives rise to Bogoliubov coefficients (apart from a convention dependent phase factor)

$$
\alpha_{D}=\left(1+\frac{i}{k \tau_{D}}-\frac{1}{2\left(k \tau_{D}\right)^{2}}\right) \quad \beta_{D}=-e^{-2 i k \tau_{D}} / 2\left(k \tau_{D}\right)^{2}
$$

where $\tau_{D}$ is a matter era conformal time at the junction. Now let $\alpha_{C}, \beta_{C}$ be the Bogoliubov coefficients calculated by continuous time integration up to the junction, then the total final Bogoliubov coefficients $\alpha_{F}, \beta_{F}$ are

$$
\alpha_{F}=\alpha_{C} \alpha_{D}+\beta_{C} \beta_{D}^{*} \quad \beta_{F}=\beta_{C} \alpha_{D}^{*}+\beta_{D} \alpha_{C}
$$

It should be noted that this last transformation $\left(\alpha_{D}, \beta_{D}\right)$ also is precisely valid independent of the magnitude of $k=2 \pi \mathrm{a} / \lambda$, just as was the transformation $\left(\alpha_{C}, \beta_{C}\right)$.

A well known result can readily be deduced from eqns (24) - (27): 


$$
|\alpha|^{2}-|\beta|^{2}=\frac{1}{2}\left(X^{*} Y+Y^{*} X\right)=\frac{i}{2 k}\left(X^{*} X^{\prime}-X^{*} X\right)
$$

and on differentiating the last expression and using eqn (26) we get zero; hence

$$
|\alpha(\tau)|^{2}-|\beta(\tau)|^{2}=\text { constant }
$$

Also, as for example in $\mathrm{AH}$, we characterize fluctuations in $\mathrm{h}_{\mathrm{ij}}$ at time $\tau$ by

$$
\Delta \mathrm{h}^{2}(\underline{\mathrm{k}}, \tau)=\frac{\mathrm{k}^{3}}{(2 \pi)^{3}} \frac{1}{2} \int \mathrm{d}^{3} \mathrm{xe} \mathrm{i}^{\mathrm{i} \cdot \mathrm{x}}\left\langle\psi\left|\mathrm{h}_{\mathrm{ij}}(\underline{\mathrm{x}}, \tau) \mathrm{h}_{\mathrm{ij}}(\mathrm{o}, \tau)\right| \psi\right\rangle
$$

where $|\psi\rangle$ is the state vector and $h_{i j}$ is given by eqn (3). (We are working in the Heisenberg representation). If then $N(\lambda, \underline{k})$ is the number of gravitons in a previous era when gravitons are defined by the operators $A(\underline{k})$ of eqn (6):

$$
\begin{gathered}
\Delta \mathrm{h}^{2}(\underline{\mathrm{k}}, \tau)=\mathrm{G}\left(\frac{\mathrm{k}}{2 \pi}\right)^{2} \sum_{\lambda=1}^{2}(\mathrm{~N}(\lambda, \underline{\mathrm{k}})+\mathrm{N}(\lambda,-\underline{\mathrm{k}})+1) \chi(\tau) \\
\chi=\left[\left(|\alpha(\tau)|^{2}+|\beta(\tau)|^{2}\right)|\xi(\underline{\mathrm{k}}, \tau)|^{2}+2 \operatorname{Re}\left(\alpha(\tau) \beta^{*}(\tau) \xi^{2}(\underline{\mathrm{k}}, \tau)\right)\right] / \mathrm{s}^{2}(\tau)
\end{gathered}
$$

where $\xi$ is the mode function of eqn (12), and the $\underline{k}$ dependence of $\alpha$ and $\beta$ has, as usual, been left implicit. We replaced $S(\tau)$ of eqn (3) by its limiting value $a(\tau)$ and then from eqn (24)

$$
\chi=|X(k, \tau)|^{2} / a^{2}(t)
$$

We now see, from eqn (26), that for small enough scale of wavelengths where $k^{2}>\mathrm{a}^{\prime \prime} / \mathrm{a}$, $\mid \mathrm{X} \mathrm{P}^{2}$ is sinusoidally fluctuating so that there is no graviton production from the corresponding era.

At this stage we can make explicit the connection with superadiabatic amplification [1] of the classical metric perturbation. Instead of the quantum mechanical expression in eqns (3) - (5) we can write classically

$$
\mathrm{h}_{\mathrm{ij}}(\underline{\mathrm{x}})=\sqrt{8 \pi \mathrm{G}} \int \frac{\mathrm{d}^{3} \mathrm{k}}{(2 \pi)^{3 / 2}} \frac{\mathrm{Z}(\mathrm{k}, \tau)}{\mathrm{a}(\tau)} \zeta_{\mathrm{ij}}(\underline{\mathrm{k}}, \underline{\mathrm{x}})
$$

where the normalized $\zeta_{\mathrm{ij}}$ and the $\mathrm{Z}$ obey respectively 


$$
\begin{aligned}
& \left(\nabla^{2}+k^{2}\right) \zeta_{i j}=0 \\
& Z^{\prime \prime}+\left(k^{2}-a^{\prime \prime} / a\right) Z=0
\end{aligned}
$$

The square of the Fourier amplitude of $h_{i j}$ is proportional to $Z^{2} / a^{2}$, which gives for suitable $a(\tau)$ superadiabatic amplification [1] of the gravitational waves, where $\mathrm{Z}$ obeys the same equation as the $X$ of eqn (46), since that is given by eqn (24). This correspondence is modified by the discrete Bogoliubov transformation of eqn (39) in cases where that is important.

The method of our paper can be readily extended to theories with a scalar component of gravity, such as Brans-Dicke theory in extended inflation [12]. For example if $\Phi(\tau)$ is the (Brans-Dicke) scalar field in extended inflation then $R \equiv a(\tau) \sqrt{ } \Phi(\tau)$ replaces $a(\tau)$ in the denominator of the $h_{i j}$ integrand [13]. Thus $R(\tau)$ replaces a $(\tau)$ in eqn (18) and in eqn (3) $S(\tau)$ are chords of the $R(\tau)$ curve. (Some illustrations of mode solutions have been given in reference 13). The case of hyper extended inflation [12], where the Brans-Dicke constant named $\omega$ is replaced by $\omega(\Phi)$, requires more extensive discussion and is postponed to a subsequent paper.

Thus the differential equations (16) and (17) become

$$
\begin{aligned}
& \alpha^{\prime}(\tau)=\frac{i}{2 k}\left(\alpha(\tau)+\beta(\tau) \mathrm{e}^{2 \mathrm{ik}\left(\tau-\tau_{0}\right)}\right) \frac{\mathrm{R}(\tau)^{\prime \prime}}{\mathrm{R}(\tau)} \\
& \beta^{\prime}(\tau)=-\frac{\mathrm{i}}{2 \mathrm{k}}\left(\beta(\tau)+\alpha(\tau) \mathrm{e}^{-2 \mathrm{ik}\left(\tau-\tau_{0}\right)}\right) \frac{\mathrm{R}(\tau)^{\prime \prime}}{\mathrm{R}(\tau)}
\end{aligned}
$$

and these can be integrated through eqn (26) with $R(\tau)$ instead of a $(\tau)$. Knowledge of the $\alpha$ and $\beta$ coefficients then gives directly the production of gravitational waves as in eqns (44) 46).

\section{Acknowledgements}

The authors acknowledge the receipt of a NATO Grant for Collaborative Research. L E Mendes acknowledges the support of a JNICT grant. RGM would like to thank Andrew Liddle for discussions. 


\section{References}

[1] L P Grishchuk, Sov. Phys. JETP 40, 409 (1975); Ann. New York Acad. Sci. 302, $439(1977)$

[2] L F Abbott and M B Wise, Nucl. Phys. B244, 541 (1984)

R Fabri and N Pollock, Phys. Lett. 125B, 189 (1982); A Starobinsky, JETP Lett. 30, 683 (1979)

[3] B Allen, Phys. Rev. D37, 2078 (1988)

[4] L F Abbott and D D Harari, Nucl. Phys. B264, 487 (1986)

[5] L Parker, Phys. Rev. 183, 1057 (1969)

[6] A B Henriques, Phys. Rev. D - to be published

[7] L H Ford and L Parker, Phys. Rev. D16, 245, 1601 (1977)

[8] N D Birrell and P C Davies, J. Phys. A13, 2109 (1980)

[9] L H Ford, Phys. Rev. D35, 2955 (1987)

[10] L Parker, Phys. Rev. Lett. 28, 705 (1972); Nature 261, 20 (1976)

[11] M R de Garcia Maia, Phys. Rev. D48, 647 (1993)

[12] D La and P J Steinhardt, Phys. Rev. Lett. 62, 376 (1989);

P J Steinhardt and E S Accetta, Phys. Rev. Lett. 64, 2740 (1990);

D La, P J Steinhardt and E W Bertschinger, Phys. Lett. B 231, 231 (1989)

R Crittenden and P J Steinhardt, Phys. Lett. B 293, 32 (1992)

F C Adams, K Freese and A H Guth, Phys. Rev. D43, 965 (1991);

E W Kolb, D S Salopek and M S Turner, Phys. Rev. D42, 3925 (1990)

[13] J D Barrow, J P Mimoso, M R de Garcia Maia, Phys. Rev. D48, 3630 (1993) 


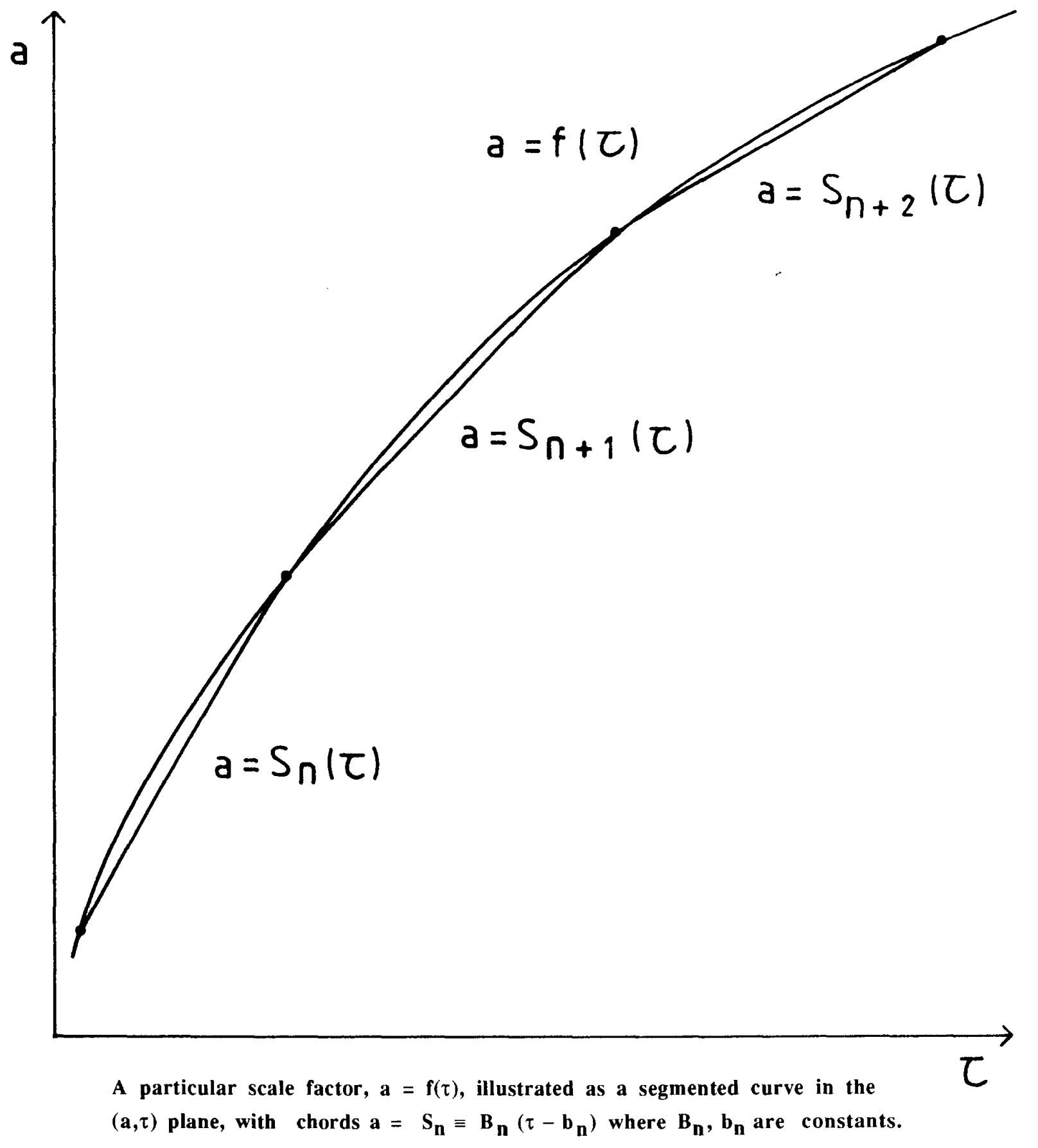

